\title{
ASSESSMENT OF OCCUPATIONAL EXPOSURE TO GASEOUS PERACETIC ACID
}

\section{STEFANO DUGHERI ${ }^{1}$, ALESSANDRO BONARI ${ }^{2}$, ILENIA POMPILIO ${ }^{2}$, MARCO COLPO ${ }^{3}$, MANFREDI MONTALTI ${ }^{2}$, NICOLA MUCCI ${ }^{2}$, and GIULIO ARCANGELI ${ }^{2}$}

${ }^{1}$ Careggi University Hospital, Florence, Italy

Industrial Hygiene and Toxicology Laboratory

${ }^{2}$ University of Florence, Florence, Italy

Department of Experimental and Clinical Medicine

${ }^{3}$ University of Florence, Florence, Italy

Department of Statistics, Informatics and Applications

\begin{abstract}
Objectives: In order to assess short-term exposure to peracetic acid (PAA) in disinfection processes, the Authors compared 4 industrial hygiene monitoring methods to evaluate their proficiency in measuring airborne PAA concentrations. Material and Methods: An active sampling by basic silica gel impregnated with methyl $p$-tolyl sulfoxide (MTSO), a passive solid phase micro-extraction technique using methyl $p$-tolyl sulfide (MTS) as on-fiber derivatization reagent, an electrochemical direct-reading PAA monitor, and a novel visual test strip PAA detector doped with 2,2'-azino-bis (3-ethylbenzothiazoline)-6-sulfonate were evaluated and tested over the range of $0.06-16 \mathrm{mg} / \mathrm{m}^{3}$, using dynamically generated PAA air concentrations. Results: The linear regression analysis of linearity and accuracy showed that the 4 methods were suitable for PAA monitoring. Peracetic acid monitoring in several use applications showed that the PAA concentration $\left(1.8 \mathrm{mg} / \mathrm{m}^{3}\right)$ was immediately dangerous to life or health as proposed by the National Institute of Occupational Safety and Health, and was frequently exceeded in wastewater treatment (up to $7.33 \mathrm{mg} / \mathrm{m}^{3}$ ), and sometimes during food and beverage processes and hospital high-level disinfection operations (up to $6.8 \mathrm{mg} / \mathrm{m}^{3}$ ). Conclusions: The methods were suitable for the quick assessment of acute exposure in PAA environmental monitoring and can assist in improving safety and air quality in the workplace where this disinfectant is used. These monitoring methods allowed the evaluation of changes to work out practices to reduce PAA vapor concentrations during the operations when workers are potentially overexposed to this strong antioxidant agent. Int J Occup Med Environ Health 2018;31(4):527-535
\end{abstract}

Key words:

Chromatography, Electrochemical sensors, Peracetic acid, Air monitoring, Short-term exposure,

Visual test strip PAA detector

\section{INTRODUCTION}

The global peracetic acid (PAA) (CAS No. 79-21-0) market is estimated to grow between 2014-2020 at a compound annual growth rate of $7.3 \%$, to reach an economic valuation of USD 652.9 million by 2020 [1]. Europe is the largest market of PAA followed by North America, and Pacific Asia. The diverse applications of the PAA may be segmented as disinfectants, sterilant, sanitizer and others. The disinfectant segment is the largest market segment worldwide; and within this segment food and beverage

Received: December 1, 2016. Accepted: November 14, 2017.

Corresponding author: Stefano Dugheri, Careggi University Hospital, Industrial Hygiene and Toxicology Laboratory, Largo Palagi 1, 50139 Florence, Italy (e-mail: stefano.dugheri@unifi.it). 
industry is the fastest growing PAA sector, accounting for over $25 \%$ of the global market, followed by healthcare, and water treatment [2]. In the pulp and paper industry, PAA has been found to be an excellent alternative for delignification and bleaching [3], though this market is still small.

Advances in manufacturing technology, the growing popularity of bio-based chemicals and innovative techniques developed for the use of PAA in many new applications have resulted in an array of products which are expected to present new opportunities for the PAA market in upcoming years.

Direct exposure to PAA may cause severe burns, allergy, and other hazardous health effects to the eyes, skin, and respiratory organs. A human study [4] has reported that exposure to $4.67 \mathrm{mg} / \mathrm{m}^{3}$ (1.55 ppm) for $12 \mathrm{~min}$ causes slight to mild irritation, and exposure to $6.23 \mathrm{mg} / \mathrm{m}^{3}$ for $60 \mathrm{~min}$ causes extreme discomfort and serious escape-impairing effects. The National Institute of Occupational Safety and Health (NIOSH) has proposed the immediately dangerous to life and health (IDLH) limit of $1.8 \mathrm{mg} / \mathrm{m}^{3}$. The American Conference of Governmental Industrial Hygienists (ACGIH) has established a threshold limit value (TLV) as a short-term exposure limit (STEL) of $1.2 \mathrm{mg} / \mathrm{m}^{3}, 15 \mathrm{~min}$ time-weighted average exposure that should not be exceeded at any time during a workday.

Currently, there are a few analytical methods for PAA vapor. These include the active [5-7] or passive [8,9] sampling methods using 2,2'-azino-bis(3-ethylbenzothiazoline)6-sulfonate (ABTS), methyl $p$-tolyl sulfide (MTS), methyl $p$-tolyl sulfoxide (MTSO), and 2-([3-\{2-[4-Amino2-(methylsulfanyl)phenyl]-1-diazenyl $\}$ phenyl]sulfonyl)1-ethanol (ADS) as reagents whether on tubes, impinger, glass fiber filters or solid phase microextraction (SPME) and later analyzed by colorimetry, liquid chromatography (LC) or gas chromatography (GC). Electrochemical sensors for PAA vapor detection are small and convenient real-time portable instruments; but experimental and field reports between direct-reading and laboratory analytical methods on reliability and accuracy are often limited.

The aim of this work has been to assess short-term exposure to airborne PAA in disinfection processes by comparing the 4 analytical methods. In addition to laboratory testing, this paper also describes the evaluation and validation protocol used for assessing PAA monitoring in food and beverage processing, wastewater treatment, and hospital high-disinfection.

\section{MATERIAL AND METHODS}

\section{Measurement devices}

Active sampling with an air flow of $1 \mathrm{l} / \mathrm{min}$ for $15 \mathrm{~min}$ was performed by MTSO basic silica gel cartridge (Giotto Biotech, Sesto Fiorentino, Italy) connected to GilAir Plus pumps (Sensydine, St. Petersburg, USA) for personal sampling, and to a 16-position automatic collector box/Bravo M Plus pump (TCR Tecora, Milano, Italy) for area sampling [7]. The cross sensitivity to hydrogen peroxide (HP) was avoided through a 37-mm cassette with quartz filter coated with titanium oxysulfate hydrate and connected to the cartridge. The cartridge was desorbed with $5 \mathrm{ml}$ of acetonitrile and the resulting solution was then made up to $10 \mathrm{ml}$ with water. The LC/ultraviolet (UV, wavelength $224 \mathrm{~nm}$ ) analysis of the methyl $p$-tolyl sulfoxide using a reversed phase Alltima C18 5 um column (250 mm length, $3 \mathrm{~mm}$ internal diameter, Grace Davison Discovery Science, Deerfield, USA) in isocratic mode (acetonitrile/water in the ratio 57/43, $1 \mathrm{ml} / \mathrm{min}$ ) was controlled with a Waters Alliance e2695.

For SPME passive sampling, the method by Pacenti et al. [9] was used with modifications. A Fast Fit Assemblies $85 \mu \mathrm{m}$ carboxen/polydimethylsiloxane (CARB/ PDMS) fiber (Supelco, Bellefonte, USA) was doped for $20 \mathrm{~s}$ in the headspace of a $10 \mathrm{ml}$ vial previous equilibrated for $20 \mathrm{~min}$ at $25^{\circ} \mathrm{C}$ and containing $5 \mu \mathrm{l}$ of MTS. Methyl $p$-tolyl sulfoxide was obtained from the reaction between PAA and MTS [10]. Personal and area sampling for $15 \mathrm{~min}$ was performed by "rapid-SPME" [11] using SPME Auto- 
matic Sampler [12] (Chromline, Prato, Italy) and a Diffusive Sampling Fiber Holder (Supelco, Bellefonte, USA), respectively. The experimental average sampling rate was $7.78 \mathrm{ml} / \mathrm{min}$. After sampling, PAA was analyzed with fast GC/mass spectrometry (MS) with a Shimadzu GC 2010/QP MS2010 series, using a narrow bore MEGA-5 MS column $(10 \mathrm{~m} \times 0.1 \mathrm{~mm} \times 0.1 \mu \mathrm{m}$ film thickness $)$. The target ion for MTSO was 138 mass-to-charge ratio.

Full automation of the LC and GC procedures was achieved using a Flex autosampler (EST Analytical, Fairfield, USA) equipped with a 45-position Multi Cartridge/ Fiber Exchange (Chromline, Prato, Italy).

Air monitoring by a continuous, direct reading detector was evaluated using a PAA Envirocell Sensor Module (ChemDAQ, Pittsburgh, USA). This monitor is a passive sampler (no pump) and the sensors are plug and play. The electrochemical sensor has a digital resolution of $0.01 \mathrm{ppm}$, minimum detection limit of $0.04 \mathrm{ppm}$ (manufacturer's specification) and a mean response time of $20 \mathrm{~s}$. Personal sampling was performed using a ChemDAQ SafeCide Portable Monitoring configurated with a tablet. The Steri-Trac Area Monitor was connected for area sampling to a management platform for data collection.

The 4th method using a novel visual test strip PAA Detector (Giotto Biotech, Sesto Fiorentino, Italy) based on reaction of ABTS to its radical cation, for sampling time of $15 \mathrm{~min}$ and quantification by a color scale to $0.4 \mathrm{ppm}[5,13,14]$. The iodide-catalyzed oxidation of the ABTS by PAA leads to the formation of a green product with 4 strong absorption maxima between $405 \mathrm{~nm}$ and $810 \mathrm{~nm}$ (highest absorbance was observed at $415 \mathrm{~nm}$ ).

\section{Dynamic calibration system}

The PAA vapor was generated by a syringe-pump Harvard Plus 11 (Harvard Apparatus, Holliston, USA), equipped with a $1 \mathrm{ml}$ gas-tight syringe set to $2 \mu \mathrm{l} / \mathrm{min}$ connected to an Adsorbent Tube Injector System (ATIS, Supelco, Bellefonte, USA). The sampling methods were evaluated using PAA atmospheres over the range of $0.06-16 \mathrm{mg} / \mathrm{m}^{3}$ (Photo 1). The 4 samplers were exposed at the same time for each PAA air concentration. The PAA vapor flow was blended with a dry air flow (1-5 1/min), and measured by a calibrated rotameter UG2.5 (Metrix Italia, Candiana Padova, Italy). The concentration of water vapor produced by the impinger, was determined by measuring the dew point temperature with a photoacoustic Multigas Monitor mod. 1312 (INNOVA, Ballerup, Denmark). Relative humidity was obtained from the dew points using the Merck Index table and the air temperature. The atmospheric pressure was determined by a digital pressure indicator Druck DPI 705 (GE Oil and Gas, Italy).

\section{Statistical analysis of method evaluation}

Robust linear regression of each method was verified in terms of linearity and accuracy by means of standard error evaluation and an independent t-test on the slope coefficient was performed. The critical $t$ value for a two-tailed test with the $\alpha$ level adopted (0.05), and the degrees of freedom for each method is presented in the Table 1. The statistical analysis was performed with Stata software v. 11.2 (Stata Corp LP. Lakeway Drive College Station, Texas, USA) and R software environment for statistical computing provided with "sandwich," "Imtest" packages [15]. Otherwise PAA detector strips were tested with the concordance correlation analysis (Cohen's $\kappa$ ) through visual evaluation from selected subjects. The instrumental limit of quantification (LLOQ) of the electrochemical sensor was provided by the manufacturer. For the chromatographic techniques, a signal-to-noise ratio of 3:1 and 10:1 was used for estimating the limit of detection (LOD) and LLOQ, respectively. The detection limit as mass/air sample volume depends on the total air volume sampled.

\section{Sampling sites}

A survey carried out in food and beverage processing, wastewater treatment, and hospital high-level disinfection 


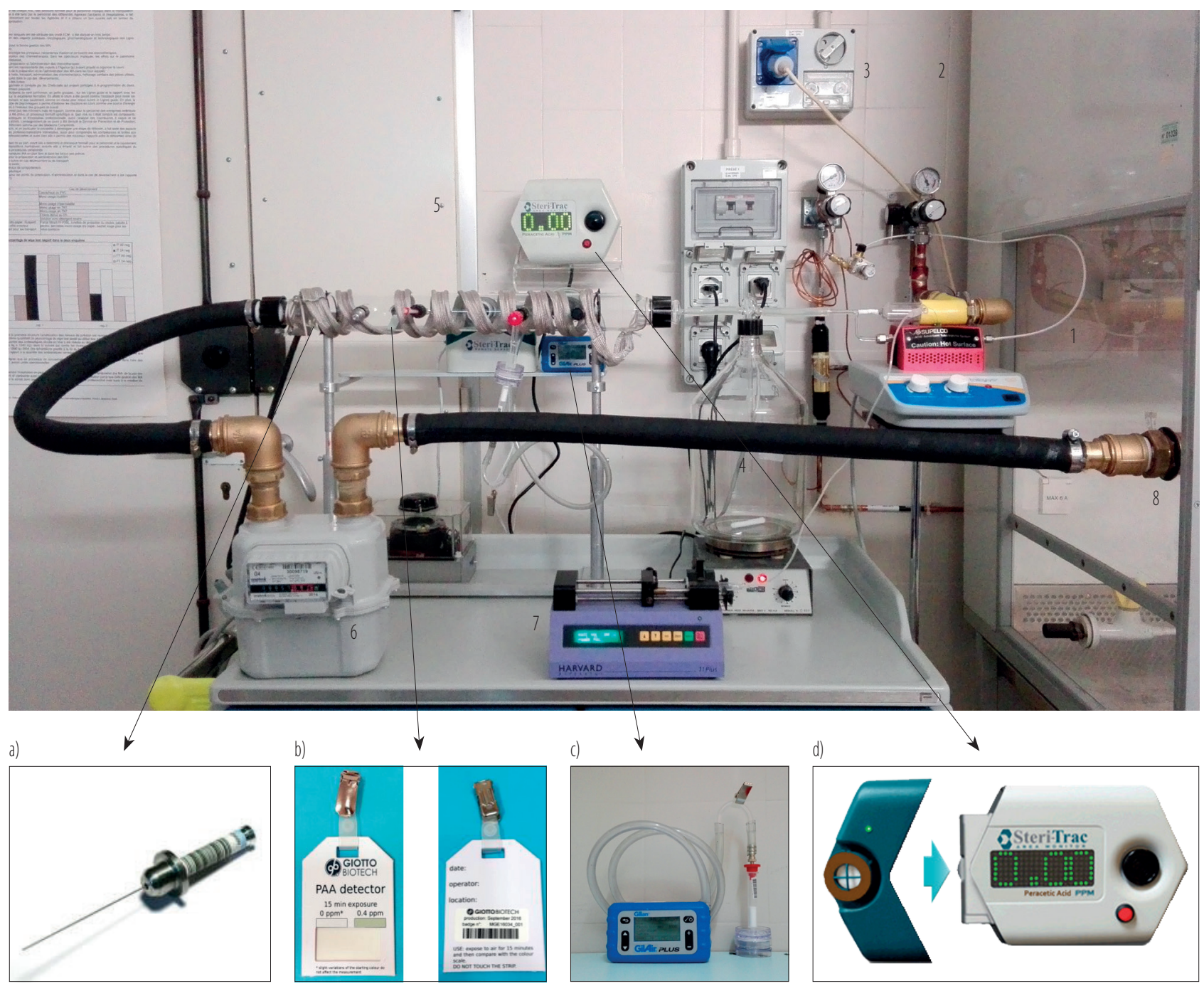

1 - thermostatic block with injector port ATIS Injector System; 2 - manometer for auxiliary gas (medical air, 1-5 1/min); 3 - manometer for inlet gas (medical air, 0.1 1/min); 4 - mixing chamber; 5 - chamber (1386 ml volume) for measurement devices: a) solid phase microextraction (SPME fiber), b) visual test strip peracetic acid (PAA) detector, c) methyl $p$-tolyl sulfoxide (MTSO) basic silica gel cartridge connected to $37 \mathrm{~mm}$ quarz fiber cassette, d) ChemDAQ electrochemical sensor; 6 - calibrated rotameter; 7 - syringe-pump; 8 - extractor hood.

Photo 1. Dynamic calibration system

department was performed during routine operations to access the risks of PAA occupational exposure. The PAA vapor was measured for various operations including:

- the replacement of PAA solution into lavaendoscopes in 32 hospital clinical units,

- filling PAA tanks for wastewater disinfection in $6 \mathrm{mu}-$ nicipal plants, monitoring the truck driver while performing his routine daily duties,
- monitoring during the maintenance of high speed filling machines (18 000 bottles/h) for soft drinks, which use the solution of $15 \%$ PAA nebulized at the maximum concentration of $2000 \mathrm{ppm}$.

A multi-data logger Babuc/A (LSI Lastem, Milano, Italy) was employed to measure temperature, relative humidity and air velocity during air sampling. 


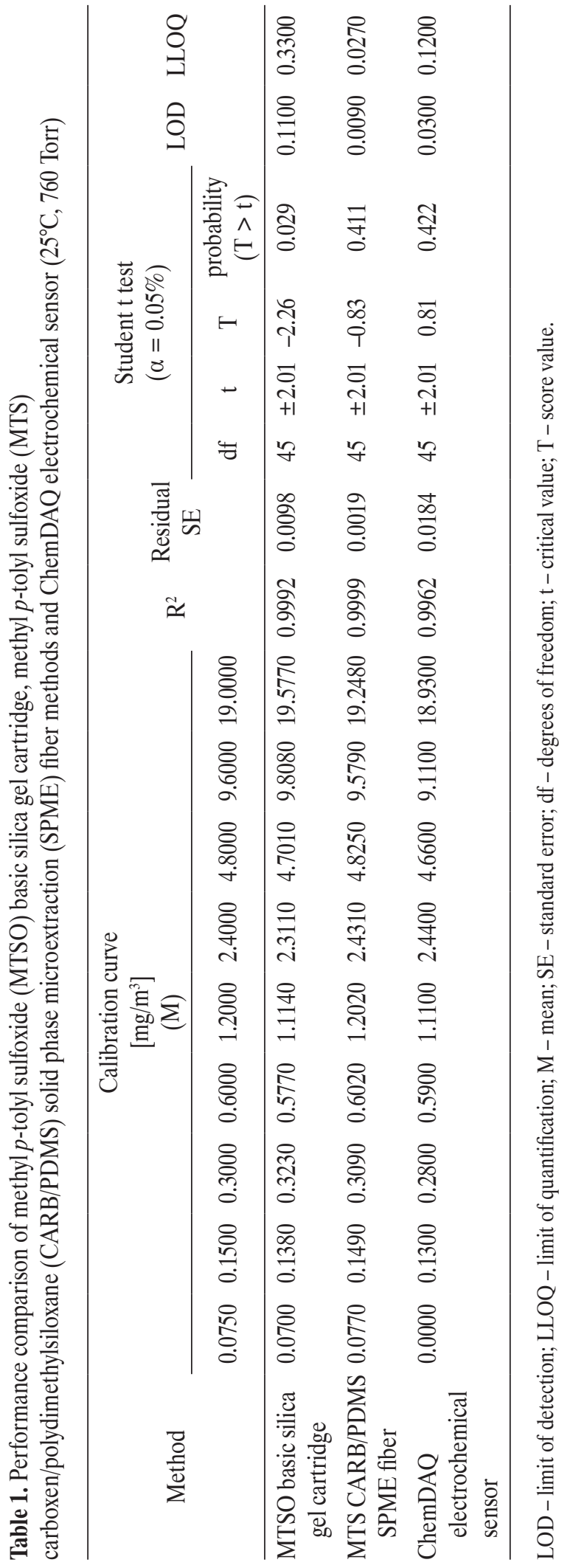

\section{RESULTS}

A comparison of the ChemDAQ electrochemical sensor, the MTSO basic silica gel cartridge and MTS CARB/ PDMS SPME fiber methods are shown in the Table 1. Independent t-test of the experimental data demonstrated that all the 3 methods compared are suitable for PAA vapor monitoring. In particular, the passive SPME technique showed the smallest variability (standard error of 0.0019) and the lowest LOD value $\left(0.027 \mathrm{mg} / \mathrm{m}^{3}\right)$. The electrochemical direct-reading instrument was sensitive to the PAA vapor concentration in terms of one order of magnitude below the TLV-STEL value. The correlation analysis of the visual test strip PAA detector showed a very good agreement level of concordance (0.8-1 Cohen's $\kappa)$ (Figure 1).

The monitoring studies showed that PAA exposures higher than 15 min time-weighted average occupational exposure limits (ACGIH $1.2 \mathrm{mg} / \mathrm{m}^{3}$ ) were found in wastewater treatment (up to $7.33 \mathrm{mg} / \mathrm{m}^{3}$ ), in food and beverage processing (up to $6.8 \mathrm{mg} / \mathrm{m}^{3}$ ) and in hospital high-level disinfection (up to $1.52 \mathrm{mg} / \mathrm{m}^{3}$ ). A detailed description of personal samplings is shown in the Table 2.

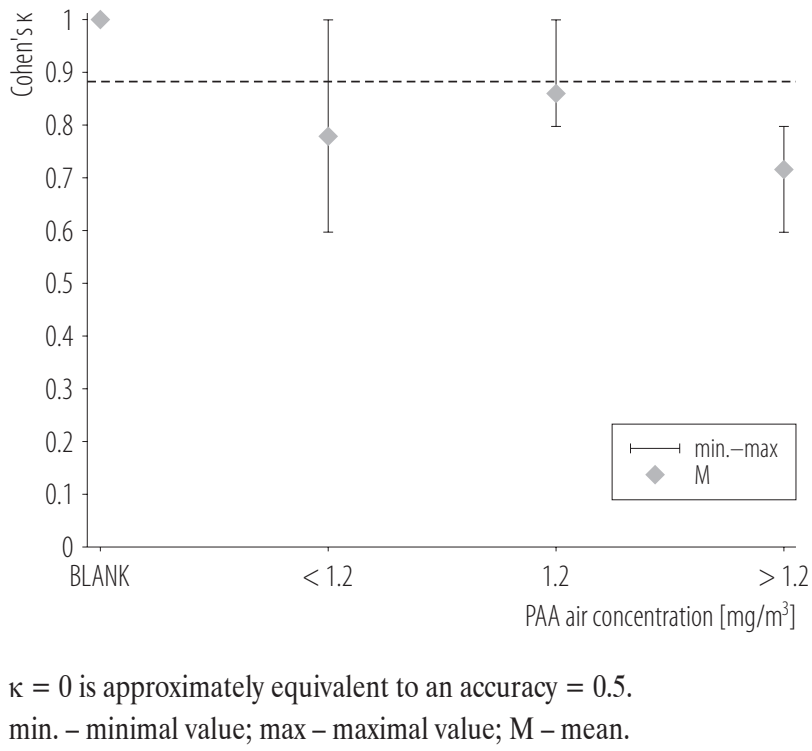

Fig. 1. Cohen's $\kappa$ vs. peracetic acid (PAA) concentration scatterplot of PAA detector strips visual evaluation testing (results of judges from selected subjects $(\mathrm{N}=11)$ ) 


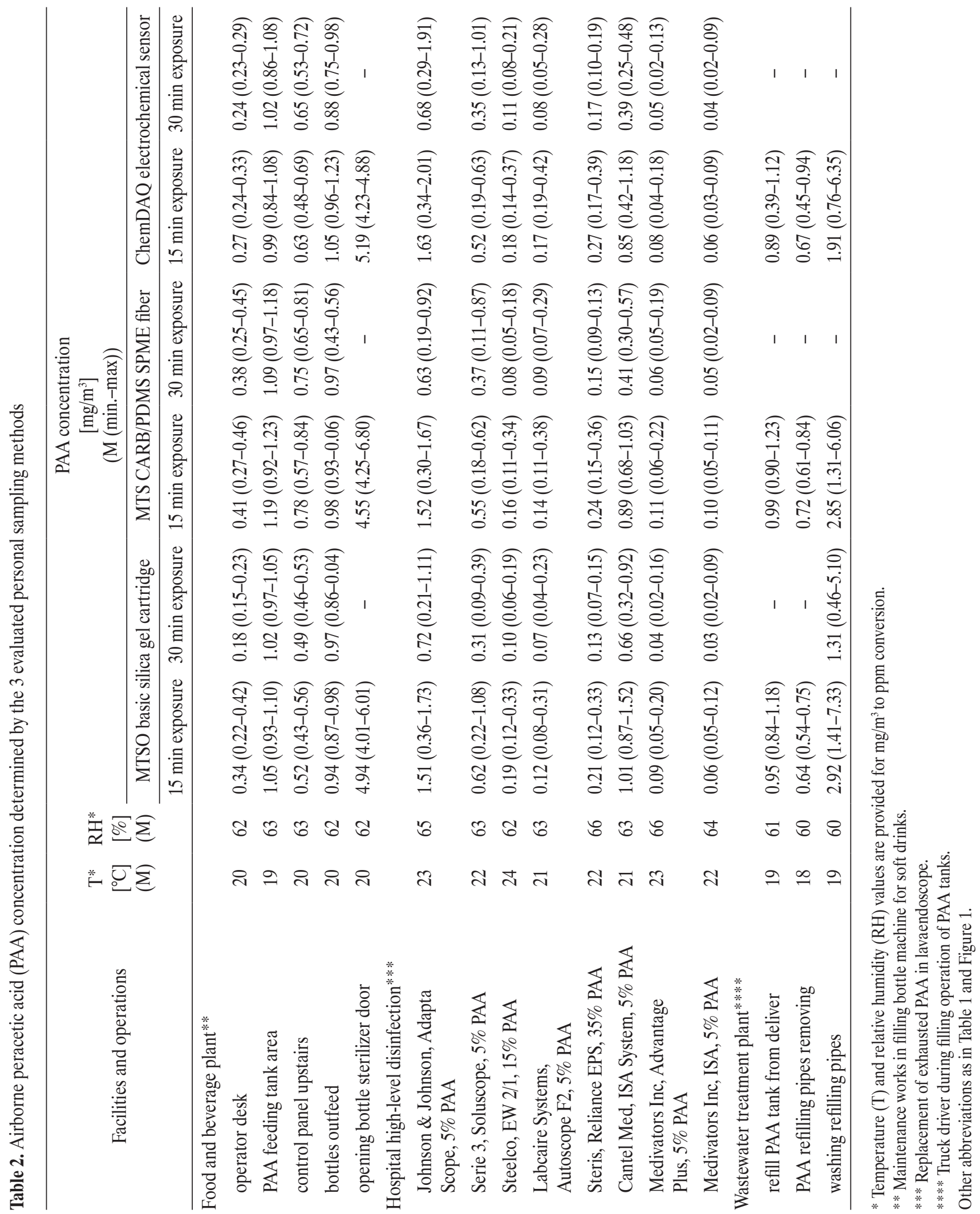




\section{DISCUSSION}

For many years the French Institute for Research and Security (Institut National de Recherche et Sécurité) had recommended the determination of airborne PAA, with TLV-time weighted average (TWA) and TLV-STEL of 0.62 and $1.56 \mathrm{mg} / \mathrm{m}^{3}$, respectively. In 2013 the ACGIH introduced a TLV-STEL value of $1.2 \mathrm{mg} / \mathrm{m}^{3}$. Considering the growing interest in this field and the increasing use of PAA in many applications, validated methods are urgently required for both expert and non-expert users. The high number of analyses necessary for the evaluation with TLV-STEL requires the use of economical and simple to use samplers, the usage of which should be as far as possible automated to avoid errors.

A disadvantage of the samplers proposed by Henneken et al. [8] and Effkermann et al. [10] results from poor storage stability, sampling periods greater than $15 \mathrm{~min}$, and furthermore they are not presently commercially available. Specifically, the first is based on the oxidation of ADS into the 2-([3-\{2-[4-Amino-2-(methylsulfoxy) phenyl]-1-diazenyl $\}$ phenyl]sulfonyl)-1-ethanol, and it has been developed with an uptake rate for PAA of $15.7 \mathrm{ml} /$ min $\pm 9.2 \%$. Since the PAA is produced from the acidcatalyzed reaction between acetic acid and HP as well as the commercial PAA formulas which are a mixture of the 3 compounds, the cross reactivity toward HP is found to be $2.45 \mathrm{ml} / \mathrm{min}$, therefore the lower flow limits the applicability of the samplers evaluated by Henneken et al. [8]. However, it has been found that for the blank system, a rapid transformation into the corresponding sulfoxide occurrs within a few days, when the reverse phase LC columns are impregnated with the ADS. In the second sampler, using an impinger for bubbling, the oxidation of MTS was measured by liquid chromatography with detection at $224 \mathrm{~nm}$ [10]. Although it was proposed by the German Research Foundation (Deutsche Forschungsgemeinschaft) [16] in 2014, this method is not particularly suited to personal sampling, and furthermore, immediately after sampling, MTS and triphenylphosphine must be added in the absorption solution.

A report [17] reviewing the market and use of biocidal products has been published recently (EU Regulation 528/2012); and in this report, a PAA active sampling method using basic silica gel impregnated with sulfoxide has been proposed [7]. In 2014, another chemical company began marketing MTSO tubes for PAA air monitoring [18].

The authors have evaluated alternative procedures that permit the instantaneous or 15 min time-weighted average sampling. Overall, these methods provide an effective assessment of occupational exposure to gaseous PAA, that may be used for assisting in improving safety and air quality in the workplace where this disinfectant is used.

The SPME passive sampler allows for automation of the sampling procedure. Thanks to structurally informative MS fragmentation patterns, the analysis by GC/MS is characterized by a higher sensitivity and better discrimination than other routine techniques employed in industrial hygiene laboratories. In addition, portable SPMEGC/MS instruments are now commercially available.

The electrochemical direct-reading instrument and the visual test strip PAA detector doped with ABTS were chosen due to their ease of use and immediate analytical results. The first portable sampler with the electrochemical sensor for PAA vapor is available with a bluetooth sensor communication, a Windows based interface for downloading file data, continuous communication with monitor displays, and connection to management platforms to generate reports and analyze historical data.

The miniaturized structure of the strip allowed real-time measure, also in terms of leak detection, inspections, and to verify any breakthrough of charcoal-impregnated face masks.

Furthermore, the 4 samplers used in this study have been simple to set up and integrate all sampling, analysis man- 
agement and software implementation into the Laboratory Information Management Systems (Bika Lab System).

Due to many limitations of the PAA toxicity database, there is still insufficient information to ascertain if a STEL alone provides adequate occupational protection. Pechacek et al. [19] suggested a TLV-TWA and STEL approach as a more appropriate one considering the potent irritation potential of PAA.

In agreement with Borak and Brosseau [20] this paper supports development of PAA Occupational Exposure Limits by means of evaluation of human exposure in several key occupational settings.

\section{CONCLUSIONS}

The experimental and field comparisons showed that the aforementioned PAA vapor measuring methods agree, and are easily integrated into an industrial hygiene plan to prevent significant acute toxicity to PAA vapor. Due to the frequent occurrence in which the TLV-STEL and IDLH values are exceeded in normal use, a method to evaluate total PAA vapor exposure is needed. Monitoring PAA vapor is especially important because of PAA's lack of biological monitoring and the similarity of the odor is mixed with acetic acid.

\section{ACKNOWLEDGMENTS}

We thank Fabrizio Niccolini of Careggi University Hospital, the sanitary director's staff, Gianluca Verdolini of Azienda USL Toscana Centro HSE Office and Marco Cacioli of Azienda USL Toscana Sud Est HSE Office who greatly assisted and supported this research. Many thanks also to Massimiliano Monti of the Clinical Engineering Service of the Hospital for the support on sterilizers market survey.

We are also immensely grateful to Tetra Laval Inc., in particular Alessandro Bonati, Barbara Botti, Roberto Ramazzini from Sidel Spa for sharing insights and experiences during on field tests that deeply improved data for this manuscript.

We would also like to show our gratitude to Simone Lippi, Fabrizio Mancuso from Ingegnerie Toscane Srl, who offered their valuable contributions in the implementation of the research in outdoor wastewater plants evaluation.

We also thank Barbara Bocking for the English review of this manuscript.

\section{REFERENCES}

1. Transparency Market Research [Internet]. Albany: The Research; 2014 [cited 2016 Nov 2]. Peracetic acid market for food and beverages, pulp and paper bleaching, water treatment, medical, agriculture and other end-users - Global industry analysis, size, share, growth, trends and forecast 2014 - 2020. Available from: http://www.transparencymarketresearch.com/peracetic-acid-market.html.

2. MarketsandMarkets [Internet]. Magarpatta city: MarketsandMarkets; 2015 [cited 2016 Nov 2]. Peracetic acid market by type (disinfectant, sanitizer, sterilant, \& others), by application (food, healthcare, water treatment, pulp \& paper, $\&$ others), by region, (North America, Europe, Asia-Pacific, \& RoW) - Global forecast to 2020. Available from: https:// www.marketsandmarkets.com/Market-Reports/peraceticacid-market-1111.html.

3. Persistence Market Research [Internet]. New York City: The Research; 2016 [cited 2016 Nov 2]. Peracetic acid market: Global industry analysis and forecast to 2015 to 2021. Available from: http://www.persistencemarketresearch.com/market-research/peracetic-acid-market.asp.

4. Fraser JAL, Thorbinson A. Fogging trials with Tenneco Organics Limited (30th June, 1986) at Collards Farm. Warrington: Solvay Interox; 1986.

5. Effkemann S, Brødsgaard S, Mortensen P, Linde SA, Karst U. Spectrophotometric and direct-reading methods for the analysis of gas phase peroxyacetic acid. Fresenius J Anal Chem. 2000;366(4):361-4.

6. Hecht G, Héry M. Generation of controlled atmospheres for the determination of the irritant potency of peroxyacetic acid. Ann Occup Hyg. 2002;46(1):89-96.

7. Hecht G, Héry M, Hubert G, Subra I. Simultaneous sampling of peroxyacetic acid and hydrogen peroxide in workplace 
atmospheres. Ann Occup Hyg. 2004;48(8):715-21, https:// doi.org/10.1093/annhyg/meh067.

8. Henneken H, Assink L, de Wit J, Vogel M, Karst U. Passive sampling of airborne peroxyacetic acid. Anal Chem. 2006;78(18):6547-55, https://doi.org/10.1021/ac060668h.

9. Pacenti M, Dugheri S, Boccalon P, Arcangeli G, Dolara P, Cupelli V. Air monitoring and assessment of occupational exposure to peracetic acid in a hospital environment. Ind Health. 2010;48(2):217-21.

10. Effkemann S, Brødsgaard S, Mortensen P, Linde SA, Karst U. Determination of gas phase peroxyacetic acid using pre-column derivatization with organic sulfide reagents and liquid chromatography. J Chromatogr A. 1999;855(2): $551-61$.

11. Augusto F, Koziel J, Pawliszyn J. Design and validation of portable SPME devices for rapid field air sampling and diffusion-based calibration. Anal Chem 2001;73:481-6.

12. Toscano P, Gioli B, Dugheri S, Salvini A, Matese A, Bonacchi A, et al. Locating industrial VOC sources with aircraft observations. Environ Pollut. 2011;159(5):1174-82, https:// doi.org/10.1016/j.envpol.2011.02.013.

13. Pinkernell U, Lüke HJ, Karst U. Selective photometric determination of peroxycarboxylic acids in the presence of hydrogen peroxide. Analyst. 1997;122:567-71, https://doi. org/10.1039/A700509A.

14. Wagner M, Brumelis D, Gehr R. Disinfection of wastewater by hydrogen peroxide or peracetic acid: Development of procedures for measurement of residual disinfectant and application to a physicochemically treated municipal effluent. Water Environ Res. 2002;74(1):33-50, https://doi. org $/ 10.2175 / 106143002 X 139730$.

15. R Core Team. R: A language and environment for statistical computing. Vienna: R Foundation for Statistical Computing; 2016.

16. Schuh C. Peroxides (peracetic acid and hydrogen peroxide). The MAK Collection for Occupational Health and Safety [Internet]. 2014;1-22 [cited 2016 Nov 2], http://doi. org/10.1002/3527600418.am7291e1713.

17. European Chemicals Agency. Regulation (EU) No. 528/2012 concerning the making available on the market and use of biocidal products. Evaluation of active substances. Assessment report [Internet]. Finland: 2015 [cited 2016 Nov 2]. Available from: http://dissemination.echa.europa.eu/Biocides/ActiveSubstances/1340-02/1340-02_Assessment_Report.pdf.

18. SKC Inc. Sampling train for hydrogen peroxide/peroxyacetic acid Cat. Nos. 225-9030 and -1345 and either Cat. No. 226193-UC or 226-199-UC - Non-agency Method 57 [Internet]. Eighty Four: SKC; 2014 [cited 2016 Nov 2]. Available from: https://www.skcinc.com/catalog/pdf/instructions/40145.pdf.

19. Pechacek N, Osorio M, Caudill J, Peterson B. Evaluation of the toxicity data for peracetic acid in deriving occupational exposure limits: A minireview. Toxicol Lett. 2015;233(1): 45-57, https://doi.org/10.1016/j.toxlet.2014.12.014.

20. Borak J, Brosseau LM. The past and future of occupational exposure limits. J Occup Environ Hyg. 2015;12(Suppl 1): S1-3, https://doi.org/10.1080/15459624.2015.1091263.

This work is available in Open Access model and licensed under a Creative Commons Attribution-NonCommercial 3.0 Poland License - http://creativecommons.org/ licenses/by-nc/3.0/pl/deed.en. 